\title{
A Case of Optic Neuritis and Transverse Myelitis in a Postpartum Ghanaian Female
}

\author{
Lanitha Srikugan, MCRP ${ }^{1}$ \\ Aathavan Loganayagam, MRCP ${ }^{2}$ \\ Kevin Kelleher, FRCP ${ }^{3}$
}

\author{
${ }^{1}$ Department of General Medicine, Darrent Valley Hospital, Kent, United Kingdom. \\ ${ }^{2}$ King's College Hospital, London, United Kingdom. \\ ${ }^{3}$ Department of General Medicine, Queen Marys Hospital, London, United Kingdom.
}

KEYWORDS: Devic's disease, Devic's syndrome, neuromyelitis optica, optic neuritis, transverse myelitis.

Rapid onset visual loss with limb involvement in a postpartum female invites a number of differential diagnoses, including infectious encephalomyelitis, vasculitis, an acute demyelinating disorder, and arterial/venous sinus thrombosis. We report an interesting, but initially puzzling, case of rapidly progressive optic neuritis and transverse myelitis in a postpartum Ghanaian female, later diagnosed as neuromyelitis optica (NMO).

\section{Case Report}

A 33-year-old Ghanaian female presented with bilateral eye pain worsened by eye movement, rapid visual loss, bifrontal headache, and right leg weakness for 48 hours. She was 8 weeks postpartum, with labor having been induced at 35 weeks due to hypertension; she was discharged on labetolol and no other medications. There was no other relevant past medical or family history, or illicit drug use.

On admission, her blood pressure was 148/99 mm Hg, pulse rate 92 beats per minute, temperature $37.8^{\circ} \mathrm{C}$, oxygen saturation $97 \%$ on room air, respiratory rate 14 breaths per minute, and Glasgow coma scale 15/15. Cranial nerve examination showed absent light perception in the right eye with a relative afferent pupillary defect, 20/30 Snellen visual acuity in the left eye, bilateral optic disc edema (worse on the right) and grade 1 hypertensive retinopathy, and slight facial asymmetry but otherwise grossly intact cranial nerves. Neurological examination of the limbs showed $5 / 5$ power in upper limbs, but flexion was weaker (3/5) than extension $(4 / 5)$ in lower limbs. There was hyperreflexia in the right arm and right leg with positive Babinski sign. Sensation, proprioception, and coordination were normal in both upper and lower limbs; a Romberg test was negative.

Other systems examinations were unremarkable. Complete blood count, standard electrolytes, erythrocyte sedimentation rate, C-reactive protein, urinalysis, chest $\mathrm{x}$-ray, and an urgent head computed tomography (CT) with contrast were all noncontributory.

To cover a broad range of possible infective processes, ceftriaxone and acyclovir were started. In view of the rapid progression of her presentation she was transferred urgently to a specialty neurology hospital. There she was started on intravenous methylprednisolone to cover possible inflam- matory causes, such as multiple sclerosis (MS) and vasculitis. However, her lower limb weakness progressed and she developed urinary retention. Lumbar puncture was performed; the opening pressure was $160 \mathrm{~mm} \mathrm{H}_{2} \mathrm{O}$. Cerebrospinal fluid (CSF) analysis demonstrated elevated protein level (121 mg/dL), 16/ $\mathrm{mm}^{3}$ lymphocytosis, normal glucose, and negative gram stain and culture. Conventional CSF polymerase chain reaction for herpes simplex, Epstein-Barr, varicella-zoster, cytomegalovirus, John Cunningham virus (to rule out progressive multifocal leukoencephalopathy), human herpesvirus 6 viruses, and toxoplasma were negative. Antinuclear antibody, anticytoplasmic antibody, antiganglioside antibodies (GM1, GQ1b), thrombophilia screen (activated protein $\mathrm{C}$ resistance ratio, lupus anticoagulant and anticardiolipin antibodies, antithrombin III, protein $\mathrm{C}$, and $\mathrm{S}$ deficiency), B12, folate, ferritin, and human immunodeficiency virus (HIV) screen were also negative. Brain magnetic resonance imaging (MRI), including venography, was normal. However, T2-weighted MRI images of the orbit showed signal hyperintensity of the left optic chiasm and optic nerves, and T2-weighted images of the spinal cord showed hyperintensity within the upper 4 and lower 4 thoracic levels. The diagnosis of NMO was entertained.

Following intravenous steroids, the patient was treated with a tapering course of oral steroids. She began to make a slow recovery with the aid of intense physiotherapy. Having walked in at her initial admission, she returned home needing crutches 4 weeks later and the visual acuity in her right eye remained 20/120 at best. Her requirement for intermittent urinary self-catheterization and rectal enemas decreased with time. She continued to be rehabilitated at home with input from the community physiotherapy team and remains in remission at 12 months.

\section{Discussion}

First described by Eugene Devic in 1894, NMO is a rare, chronic inflammatory, demyelinating disease of the central nervous system. ${ }^{1}$ It is more common among Asians and Africans, with a female predominance (females comprise over two-thirds of patients and more than $80 \%$ of those with the relapsing form of the disease). ${ }^{2}$ The mean age of onset is 35 to 47 years. $^{2}$ The prevalence and incidence of 
NMO has not been established, partly because the disease is underrecognized and often confused with MS.

\section{Pathophysiology and Clinical Features}

NMO is a nonhereditary autoimmune condition distinct from MS. It is characterized by antibodies (NMO-immunoglobulin G [IgG]) against aquaporin 4 in myelin within astrocytes, which form part of the blood-brain barrier. ${ }^{2}$ The damage in the spinal cord can range from inflammatory demyelination to necrosis. The inflammatory lesions in NMO have been classified as type II lesions (complement-mediated demyelination) - they differ from MS in their prominent perivascular distribution.

NMO presents with attacks of acute optic neuritis, usually bilateral, associated with severe transverse myelopathy occurring at the same time as or following the optic neuritis within a few days, weeks, or occasionally months. The following characteristics help to differentiate NMO from $\mathrm{MS}^{3}$ :

- The presence of transverse myelitis, bilateral neurological signs, and longitudinal extensive necrotic lesions on spine MRI point toward NMO.

- Clinical involvement beyond the spinal cord and optic nerves, presence of oligoclonal bands in CSF, white matter lesions on brain MRI, and multiple small peripheral lesions on spine MRI point toward MS.

- Acute severe presentation and rapid deterioration of neurological signs are more likely with NMO.

\section{Diagnosis}

Revised criteria proposed by the Mayo Clinic for diagnosis of NMO require 2 "absolute criteria" plus at least 2 "supportive criteria" 4 :

Absolute criteria:

- Optic neuritis.

- Acute myelitis.

Supportive criteria:

- Brain MRI not meeting criteria for MS at disease onset.

- Spinal cord MRI with contiguous T2-weighted signal abnormality extending over 3 or more vertebral segments, indicating a relatively large lesion in the spinal cord.

- NMO-IgG seropositive status.

The criteria incorporate the recently developed NMO-IgG test, which is currently available through centers such as the Mayo Clinic (Minnesota) and John Radcliffe Hospital (Oxford, United Kingdom). Our patient was not tested for NMO-IgG when the assay became available because it is not known whether the test may be falsely negative during remission.

\section{Management}

Unlike MS, NMO rarely has a secondary progressive phase in which patients have increasing neurologic decline between attacks without remission. Instead, disabilities arise from the acute attacks. ${ }^{2}$ NMO may follow 1 of 3 courses:

- Monophasic (single episode with permanent remission; approximately $20 \%$ of patients have permanent visual loss and $30 \%$ have permanent paralysis in 1 or both legs).

- Relapsing (at least $85 \%$ of cases are characterized by 1 or more relapses, which usually occur early, with about $55 \%$ of patients having a relapse in the first year and $90 \%$ in the first 5 years). ${ }^{2}$

- Progressive downhill course to death during first attack. Recovery is typically poor, possibly due to necrosis, with an estimated $50 \%$ death rate. ${ }^{1}$

Little is known about the effect of pregnancy on the disease progression of NMO. There are 3 other case reports of first presentation of NMO during pregnancy. In 1 case the patient was a known systemic lupus erythematosus (SLE) sufferer and NMO relapsed postpartum. ${ }^{5}$

In the acute phase, high-dose intravenous steroid and plasmapheresis are useful. No controlled trials have established the effectiveness of treatments for the prevention of attacks. There is often steroid-dependence, meaning that relapses are not uncommon during steroid withdrawal. Long-term immunosuppressants are required to reduce the frequency and severity of attacks; these include azathioprine plus prednisone, mycophenolate mofetil plus prednisone, mitoxantrone, and cyclophosphamide. Rituximab, a monoclonal antibody, is currently under study. There are also case reports of monthly maintenance doses of intravenous gamma globulin resulting in prevention of relapses at 12 months. ${ }^{6}$

\section{Address for correspondence and reprint requests:}

Kevin Kelleher, FRCP, Department of General Medicine, Queen

Mary's Hospital Sidcup, Kent, DA14 6LT, United Kingdom; Telephone: 02083022678 ext 4750; E-mail:

kevin.kelleher@qms.nhs.uk Received 13 January 2008; revision received 25 April 2008; accepted 14 May 2008.

\section{References}

1. De Seze J. Neuromyelitis optica. Arch Neurol. 2003;60(9):1336.

2. Wingerchuk DM. Neuromyelitis optica. Int MS J. 2006;13(2):42-50.

3. Weinshenker BG. Neuromyelitis optica: what it is and what it might be. Lancet. 2003;361(9361):889-890.

4. Wingerchuk DM, Lennon VA, Pittock SJ, Lucchinetti CF, Weinshenker BG. Revised diagnostic criteria for neuromyelitis optica. Neurology. 2006;66: 1485-1489.

5. Bonnet F, Mercié P, Morlat P, et al. Devic's neuromyelitis optica during pregnancy in a patient with systemic lupus erythematosus. Lupus. 1999; 8(3):244-247.

6. Bakker J, Metz L. Devic's neuromyelitis optica treated with intravenous gamma globulin. Can J Neurol Sci. 2004;31(2):265-267. 